\title{
Menopausal transition and postmenopausal health problems: a review on its bio-cultural perspectives
}

\author{
Shailendra Kumar Mishra
}

Department of Anthropology, Guru Ghasidas Vishwavidyalaya, Bilaspur, India; shailendra17@gmail.com

Received 29 December 2011; revised 18 January 2011; accepted 17 March 2011.

\begin{abstract}
This paper documents health problems faced by menopausal and postmenopausal women. These women constitute a sizable proportion of India's population though there exist no health care programmes to cater their specific health needs and health vulnerabilities arose due to menopausal transition. Researchers have generally tried to determine age at menopause and a few have explored psychosomatic problems experienced by them. Abrupt changes in hormone levels among women generally bring out several physical infirmities which have hardly been the interest of research. Studies on menopausal and postmenopausal health problems and their bio-cultural correlates are warranted. Efforts should be made to understand the process of aging among women in relation to menopausal transition. Cross-cultural differences in coping mechanisms to minimise the health problems arose due to menopausal transition are important to study. This review argues provision of culturally appropriate health care programs to facilitate easier menopausal transition and to ensure healthy postmenopausal life for women.
\end{abstract}

Keywords: Postmenopausal Health; Menopausal Transition; Biocultural Perspectives; India

\section{INTRODUCTION}

Women's health has been a global concern for many decades $[1,2]$. The focus of women's health researchers and health policy planners has also shifted towards postmenopausal women since recent trends suggest an increase in their number and life expectancy $[3,4]$. A total of 130 million Indian women are expected to live beyond menopause by 2015. Under current demographic trends, menopausal and postmenopausal health has emerged as an important public health concern in India owing to improved economic conditions, rapid lifestyle changes, and increased longevity [5]. Generally, women have more complex and stressful aging process as men do, as a consequence of hormonal changes that occur during menopausal transition [6]. The onset of this physiological development not only marks the end of women' reproductive function but makes them more vulnerable to a new set of health problems including cardiovascular diseases, osteoporosis and so on [7].

Menopause (as defined by the World Health Organization) is the permanent cessation of menstruation due to loss of ovarian follicular activity [8]. This definition uses both, a symptom that can be identified by a woman (the end of menstruation) and a sign that can be measured (loss of follicular activities resulting in changes in levels of hormones). There remain, however, shortcomings in this definition. First, follicular activity can continue even in the absence of menstruation, for example, in case of hysterectomy, ovaries may remain functional. Second, follicular activity can end, but menstruation can continue through the use of cyclic hormonal therapy. Third drawback of this definition is how women experience menopause, which may vary within and between the social groups. Most women perceive menopause to be a marker for the end of childbearing. The end of menstruation can, therefore, be an emotional event. Some women may react to the cessation of menstruation with relief (no more birth control); other may describe deep sadness because they can no longer bear children.

Researchers and health care providers have generally agreed to define menopause as the last menstrual period followed by at least twelve months of amenorrhea (no menstrual bleeding). The advantage of this definition is that it identifies a single, measurable variable within this period of transition. The definition also enables to compute median and mean age at menopause for inter- and intra-population comparisons. Although last menstrual period is a clinically useful marker of an event, the average woman's sense of the process of the menopausal transition is better described by the term 'perimeno- 
pause', a time period during which a women wonders if each period of bleeding is the last. Various staging system have been proposed to differentiate premenopausal (regular cycling) from perimenopausal (irregular cycling) and postmenopausal (no cycle from last twelve months) ranging from three to thirteen categories of menopausal status $[9,10]$.

As noted, the perimenopause is frequently accompanied by symptoms of varying intensity that are believed to reflect marked fluctuations in levels of estrogen and progesterone or its outright deficiency [11]. The tissues that are most affected by reduced estrogen level are the ovaries, uterus, vagina, breast, and urinary tract. Tissues such as the hypothalamus, skin, cardiovascular tissues, and bones may also be affected [12].

\section{EFFECTS OF MENOPAUSE ON OVERALL HEALTH}

For centuries, disturbances of mood and behaviour have been associated with reproductive endocrine system change in women. Much of the current understanding of these disorders is based on myths, unwarranted assumptions and conclusions derived from poorly constructed studies [2]. Our understanding of relationships among aging, the menopause and behavioural change is inadequate. Many studies have relied on small samples of self-selected women seeking treatment for symptoms. As a result, the actual prevalence of minor psychological and somatic symptoms directly related to lowered levels of ovarian estrogen remains speculative at best.

Long-term consequences of changes in ovarian hormonal levels includes morbidities associated with aging such as cardiovascular diseases, osteoporosis, problems related to memorization, urinary incontinence, skin aging and so on [13-17]. Postmenopausal women are generally disproportionately affected by osteoporosis and fracture rates among women are approximately twice as high as men. The cause of osteoporosis is very complex but it is clear that hormonal changes after menopause increase the rate of bone resorption, leading to greater risk of osteoporosis. Brain is also a target for estrogen and other gonadal steroids. Subsets of neurons possess intranuclear receptors for estrogen. Moreover, it is found that problems such Alzheimer's disease expressed earlier in women than in men [15]. This may be related to estrogen loss that occurs with menopause. The incidence of coronary heart disease (CHD) is extremely rare in premenopausal women, even in high-risk population, and much lower in perimenopausal women than in men of similar age. However, the incidence rapidly increases in women after menopause and loss of ovarian function. Some of the studies show that women who experienced early menopause have increased risk of heart diseases. A cohort study of postmenopausal women, age 50-65 years at enrollment and followed up to 10 years, showed that the risk of cardiovascular mortality was higher for women with early menopause than those with late menopause [18].

Urogenital problems are experienced by one-third of women from age of fifty years and onward [19]. Urinary inconsistence is one of the most significant urogenital disorders. Female's lower urinary tract is a target organ for the action of the sex steroid hormones estrogen and progesterone since estrogen receptors are found in the urethra and lower urinary tract [20]. Skin aging has also been reported to be affected by the reduction of female hormones after menopause [21]. Carbohydrate metabolism and adipose tissue distribution are also regulated by female sex hormones, and metabolic change leads to obesity [22,23].

\section{MENOPAUSE AND BIO-CULTURAL PERSPECTIVE}

The biology and culture can be brought together into a comprehensive study of human life events in myriad ways. The holism of the bio-cultural framework allows anthropologists to explore a wide range of research topics, across any length of time, using different measures of fitness to understand how biology, culture and environment interact to shape a particular phenomenon. In addition, the bio-cultural perspective examines the developmental and environmental processes that bring about human variations [24,25] for example, in menopausal symptoms. Thus, bio-cultural approach is a heuristic tool to understand the ways in which culture, biology and the environment interact in determining an aspect of human variation. To study reproductive health problems and its management in a population, scholars have emphasized on the role of 'local biology' refers the ways in which physiological processes shape and are shaped by the socio-cultural milieu in which they are embedded [26-28]. Dressler and his colleagues have also contributed to an understanding of health in relation to 'cultural consonance'- a measure of how closely an individual's behaviour approximates the 'guiding sensibilities' of his or her own culture in relation to lifestyle and social support norms [29]. Researchers have also focused on cultural variables such as social support or social power in relation to physiological outcomes. The measurable social parameters which can affect menopause include medical interventions, attitudes about aging, birth control policy, smoking practices, diet preferences, patterns of breastfeeding, and the socially appropriate timing of motherhood.

The bio-cultural perspectives provides a way to address simultaneously many different questions raised 
regarding variation in age at menopause, variation in symptoms associated with menopause, and why postmenopausal women have a comparatively squeal life with high burden of diseases and disabilities [8]. A bio-cultural perspective allows us to consider the ways in which social values and biomedicine complicate the relationship between end of fertility and cessation of menses as well as its repercussions on postmenopausal life.

\section{MENOPAUSE AND CROSS-CULTURAL RESEARCH}

Age at menopause is studied as the outcome of a process of follicle loss - whether the depletion of follicles occurs at age thirty five or fifty five. Fifty is the most commonly reported age at menopause, and more women experienced menopause before the age of fifty than after [30,31]. Although the average age of onset of puberty has decreased over time, there is no indication of a relationship between a women's age at menarche and the timing of the menopause [12]. Early menopause is found to be associated with certain lifestyle factors like smoking, but the exact nature of this effect remains speculative [30]. Also, women who have never had children tend to experience an earlier menopause [32]. If the menopause occurs before age forty years, as it does in roughly eight percent of women, it is considered 'premature' ovarian failure, not menopause. The emphasis on numerical measurement leads to the establishment of a cut-off between 'premature' (generally before age forty years) and a 'normal' age at menopause. Contemporary cross-cultural and historical studies demonstrate further that the cut-offs imposed on human variation to differentiate 'normal' from pathological are culture-bound and arbitrary. Several caveats are in order, however. First, human variation is often visible and generally measurable. In other words, while the cut-off is medically established, the existence of human biological variation is not a cultural construct. Second, there are significant health consequences associated with some forms of human variation. Moreover, sometimes it is noted that the norms established for one population may not be exactly applicable in other populations.

Most descriptions on menopausal transition and postmenopausal health rely on clinical impressions or on small sample of women selected from patient populations rather than from general public [33]. As a result, the extent to which a woman suffers from symptoms of menopause and the risks of developing diseases and disabilities during postmenopausal life remains unclear. The menopause elicits a variety of societal responses, the specifics of which depend on a woman's particular cultural milieu. Besides variations in cultural cognizance and recognition of the menopause, anthropologists have observed different effects on the role of the women ranging from an increase in freedom and status to the complete loss of the role [34].

\section{MENOPAUSE STUDIES: INDIAN SCENARIO}

In India, studies have mainly been carried out to determine the age at menopause in various population groups [35-38] and symptoms experienced by them during menopause $[39,40]$. Under the leadership of Pieter Vann Keep a series of surveys were conducted in northern India but the interest in the relationship between culture and menopausal age and subjective reporting of symptoms remained central in those [27]. Only a few studies have been undertaken to understand the effects of menopausal transition in relation to aging process on general health profile of women in postmenopausal life $[41,42]$. Added to this, a few studies are available based on clinical sample which have investigated effects of hormone replacement therapy on postmenopausal women's health $[43,44]$. The studies carried out among different population groups of India suggest lower age at menopause as compared to women of western countries $[35,36,39]$. The factors found to be responsible for lower age at menopause include socioeconomic status, poor nutrition, reproductive history and health care ignorance apart from genetic predisposition which is hard to establish $[42,45]$.

\section{FUTURE PROSPECTS AND KEY RECOMMENDATIONS}

The cohort of postmenopausal women is increasing in India. The abrupt endocrine changes during menopausal transition have important impacts on the physiology of female body which exacerbate risks for many diseases and disabilities during postmenopausal life. Further, life expectancy is higher for females than males across the globe including India. Thus, in general, females lead a longer but squeal postmenopausal life in the countries like India.

India is a vast country with variations in terms of ecology, ethnicity, socioeconomic status, cultural norms, social values, as well as distribution, availability and accessibility of health care resources. People across the country in general and in urban areas in particular are exposed to the forces of modernization which have strong impact on their lifestyle, health status and health care practices. Acknowledging the broad resemblances, differences are expected in problems experienced during menopause and morbidity profile of females during postmenopausal life due to differences in local physical 
environment and also as an effect of different microcultural factors. However, cross-cultural studies of menopause are sparse, and the previous studies have mainly dealt with variations in age at menopause and symptoms related to menopause. Efforts have hardly been made to study the health problems faced by women after menopause which are largely the results of drastic hormonal changes occur during menopause and generally not the results of normal process of aging.

The studies are needed to investigate physical, physiological and social changes experienced during the menopausal transitions along with the nature and magnitude of health problems during postmenopausal life among women living in different ecological niches. It would be worthwhile to focus on bio-cultural correlates of these variations. It is important to assess the level of awareness, preparedness and management strategies of menopause, the prevalence and magnitude of both physical and mental health problems instigated or exacerbated by menopausal transition among women. The researchers should identify the roles of social institutions and health care services in facilitating women an easy menopausal transition and management of postmenopausal health problems as well as the variations in cultural construct of menopause in different cultural settings and how far the cultural norms help mitigating the problems during menopausal transition. The studies should provide exhaustive account of women's views, health care practices, and their expectations about roles of social institutions to tackle normal menopausal transition and healthy postmenopausal life. Such studies will help devising culturally appropriate public health programs for menopausal and postmenopausal women to ensure healthy post-reproductive life for them.

\section{REFERENCES}

[1] United Nations (1995) The world's women, 1995: trends and statistics. (Social Statistics and Indicators, Series K, No.12) New York: United States.

[2] World Health Organization (1996) Research on the menopause in the 1990s (Report of a WHO scientific group, WHO Technical Report Series, 886). Geneva: World Health Organization.

[3] World Health Report (1998) Life in the 21st century: A vision for all. Geneva: World Health Organization.

[4] World Health Organization (2000) Women aging and health. Fact sheet No. 252. Geneva: WHO.

[5] Sengupta, A. (2003) The emergence of menopause in India. Climacteric, 6, 92-95.

[6] Morrison, J.H., Brinton, R.D., Schmidt, P.J. and Gore, A.C. (2006) Estrogen, menopause, and the aging brain: How neuroscience can inform hormone therapy in women. The Journal of Neuroscience, 26, 10332-10348. doi:10.1523/JNEUROSCI.3369-06.2006

[7] Shakhatreh, F.M. and Mas'ad, D. (2006) Menopausal symptoms and health problems of women aged 50-65 years in Southern Jordan. Climacteric, 9, 305-311. doi:10.1080/13697130600861542

[8] World Health Organization (WHO). (1981) Research on the Menopause. WHO Technical Report Series No.670. Geneva: World Health Organization.

[9] Oldenhave, A., Jaszmann, L.J.B., Haspel, A.A. and Everaerd, W.T.A.M. (1993) Impact of climacteric on wellbeing: A survey based on 5213 women 39 to 60 years old. American Journal of Obstetrics and Gynecology, 168, 772-780.

[10] Punyahotra, S., Dennerstein, L. and Lehert, P. (1997) Menopausal experiences of Thai women, Part I: Symptom and their correlates. Maturitas, 26, 1-7. doi:10.1016/S0378-5122(96)01058-4

[11] Upton, G.V. (1982) The perimenopause: Physiological correlates and clinical management. Journal of Reproductive Medicine, 27, 1-27.

[12] Utian, W.H. (1989) Biosynthesis and physiologic effects of estrogen deficiency: A review. American Journal of Obstetric and Gynecology, 161, 1828-1831.

[13] Jorm, A.F., Korten, A.E. and Henderson, A.S. (1987) The prevalence of dementia: A quantitative integration of the literature. Acta Pyschiatrica Scandinavica, 76, 465-497. doi:10.1111/j.1600-0447.1987.tb02906.x

[14] Isles, C.G., Hole, D.J., Hawthrone, V.M. and Lever, A.F. (1992) Relation between coronary mortality in women of the Renfrew and Paisley survey: Comparison with men. Lancet, 339, 702-706. doi:10.1016/0140-6736(92)90599-X

[15] Kokmen, E., Beard, C.M., O’Brien, P.C. and Kurland, L.T. (1996) Epidemiology of dementia in Rochester, Minnesota. Mayo Clinic Proceedings, 71, 275-282. doi:10.4065/71.3.275

[16] Messina, M., Ho, S. and Alekel, D.L. (2004) Skeletal benefits of soy isoflavons: A review of the clinical trail and epidemiologic data. Current Opinion in Clinical $\mathrm{Nu}$ trition and Metabolic Care, 7, 649-658. doi:10.1097/00075197-200411000-00010

[17] Genazzani, A.R., Gambacciani, M., Schneider, H.P. and Christiansen, C. (2005) International Menopause Society Expert Workshop. Postmenopausal osteoporosis: Therapeutic options. Climacteric, 8, 99-109.

[18] van der Schouw, Y.T., van der Graaf, Y., Steyerberg, E.W., Eijekemans, J.C. and Banga, J.D. (1996) Age at menopause as a risk factor for cardiovascular mortality. Lancet, 347, 714-718. doi:10.1016/S0140-6736(96)90075-6

[19] Samsioe, G. (1998) Urogenital aging - A hidden problem. American Journal of Obstetric and Gynecology, 178, S245-249. doi:10.1016/S0002-9378(98)70555-1

[20] Iosif, S., Henriksson, L. and Ulmsten, U. (1981) The frequency disorders of the lower urinary tract, urinary incontinence in particular, as evaluated by a questionnaire survey in a gynecological health control population. Acta Obstetricia et Gynecologica Scandinavica, 60, 71-76. doi:10.3109/00016348109154113

[21] Raine-Fenning, N. J., Brincat, M. P. and Muscat-Baron, Y. (2003) Skin aging and menopause: Implications for treatment. American Journal of Clinical Dermatology, 4, 371-378. doi:10.2165/00128071-200304060-00001

[22] Davidson, M.B. (1979) The effect of aging on carbohydrate metabolism: A review of the English literature and 
a practical approach to the diagnosis of diabetes mellitus in the elderly. Metabolism, 28, 688-705.

doi:10.1016/0026-0495(79)90024-6

[23] Björntorp, P. (1996) The regulation of adipose tissue distribution in humans. International Journal of Obesity Related Metabolic Disorders, 20, 291-302.

[24] Bogin, B. and Smith, B.H. (1996) Evolution of the human life cycle. American Journal of Human Biology, 8, 703-716.

doi:10.1002/(SICI)1520-6300(1996)8:6<703::AID-AJH B2>3.0.CO;2-U

[25] Worthman, C.M. (1993) Biocultural interactions in human development. In: ME Pereira, LA Fairbanks (eds). Juvenile Primates: Life History, Development and Behaviour. New York: Oxford University Press. 339-358.

[26] Hinton, A.L. (1999) Introduction: Developing a biocultural approach to the emotions. In: AL Hinton (ed). Biocultural Approaches to the Emotions. New York: Cambridge University Press. 1-37.

[27] Melby, M.K., Lock, M. and Kaufert, P. (2005) Culture and symptom reporting at menopause. Human Reproduction Update, 11, 495-512. doi:10.1093/humupd/dmi018

[28] Worthman C.M. and Kohrt, B. (2005) Receding horizons of health: Biocultural approaches to public health paradoxes. Social Science and Medicine, 61, 861-878. doi:10.1016/j.socscimed.2004.08.052

[29] Dressler, W.W. and Bindon, J.R. (2000) The health consequences of cultural consonance: Cultural dimensions of lifestyle, social support, and arterial blood pressure in an African American community. American Anthropologist, 102, 244-260. doi:10.1525/aa.2000.102.2.244

[30] Brambilla, D. and McKinlay, S.M. (1989) A prospective study of factors affecting age at menopause. Journal of Clinical Epidemiology, 42, 1031-1039. doi:10.1016/0895-4356(89)90044-9

[31] Whelan, E.A., Sandler, D.P., McConnaughey, D.R. and Weinberg, C. (1990) Menstrual and reproductive characteristics and age at natural menopause. American Journal of Epidemiology, 131, 625-632.

[32] McKinlay, S.M., Brambilla, D.J. and Posner, J.G. (1992) The normal menopause transition. Journal of Human Biology, 4, 37-46. doi:10.1002/ajhb.1310040107

[33] Stotland, N.L. (2005) The context of midlife in women. In: DE Stewart (ed). Menopause: A Mental Health Practitioner's Guide. Washington DC: American Psychiatric Publishing, Inc. 1-14.
[34] Utian, W.H. (1997) Menopause - A modern perspective from a controversial history. Maturitas, 26, 73-82. doi:10.1016/S0378-5122(96)01092-4

[35] Piplai, C. (1991) Age at menopause of Tamang women tea-labourers of Jalpaiguri district, West Bengal, India. International Journal of Anthropology, 6, 233-236. doi:10.1007/BF02444059

[36] Kriplani, A. and Banerjee, K. (2005) An overview of age of onset of menopause in northern India. Maturitas, 52, 199-204. doi:10.1016/j.maturitas.2005.02.001

[37] Sharma, N., Vaid, S. and Manhas, A. (2005) Age at menopause in two caste group (Brahmin and Rajputs) from rural areas of Jammu. Anthropologist, 7, 111-113.

[38] Baghla, N. and Sharma, S. (2008) Onset age of menopause among women in Kangra district of Himachal Pradesh. Anthropologist, 10, 305-307.

[39] Singh, A. and Arora, A.K. (2005) Profile of menopausal women in rural north India. Climacteric, 8, 177-184. doi:10.1080/13697130500117920

[40] Kapur, P., Sinha, B. and Pereira, B. (2009) Measuring climacteric symptom and age at natural menopause in an Indian population using the Greene Climacteric Scale. Menopause, 16, 378-384. doi:10.1097/gme.0b013e31818a2be9

[41] Nongkynrih, B. (2004) The prevalence of fragility fractures amongst post-menopausal women in rural Haryana: a community based study. Indian Journal of Preventive and Social Medicine, 35, 147-155.

[42] Bairy, L., Adiga, S., Bhat, P. and Bhat, R. (2009) Prevalence of menopause symptom and quality of life after menopause in women form South India. Australian and New Zealand Journal of Obstetrics and Gynecology, 49, 106-109. doi:10.1111/j.1479-828X.2009.00955.x

[43] Naddaf, A. and Semreen, M. (2005) Influences of postmenopausal hormone replacement therapy on women's health. Pakistan Journal of Biological Sciences, 8, 198-201.

[44] Datta, A.K., Sundarka, A., Sundarka, M.K. and Shankar, P. (2001) Female hormone replacement therapy in postmenopausal women: where are we today? Journal of Indian Academy of Clinical Medicine, 2, 298-304.

[45] Mahadevan, K., Murthy, M.S.R., Reddy, P.R. and Bhaskaran, S. (1982) Early menopause and its determinants. Journal of Biosocial Science, 14, 473-479. doi:10.1017/S0021932000014334 